\section{Standard Work Specifications for Single-Family Home Energy Upgrades Summary}

The U.S. Department of Energy (DOE), the National Renewable Energy Laboratory (NREL) and numerous industry stakeholders developed the Standard Work Specifications for Single-Family Home Energy Upgrades to define the minimum requirements for highquality residential energy upgrades. The Standard Work Specifications for Single-Family Home Energy Upgrades is the first of three documents that will be published in 2012 and 2013 as part of the Guidelines for Home Energy Professionals project. Specifications for manufactured housing and multifamily homes will also be available.

DOE, NREL, and industry developed the Standard Work Specifications under the Weatherization Assistance Program, building on more than 30 years of experience performing millions of home energy efficiency upgrades. Over the years, weatherization technicians and program administrators identified a need for documentation listing the technical requirements for the energy upgrades carried out under the efforts of the program.

Today, the Standard Work Specifications provide a unique source for defining high-quality home energy upgrades, establishing clear expectations for homeowners, contractors, trainers, workers, program administrators, and organizations that provide financing for energy upgrades.

"The standard work specifications are a bold and important step for our industry — to build consistency and accuracy for homeowners and investors alike!"

- Amy Fazio, Chief Executive Officer at Affordable Comfort Inc. (ACl)

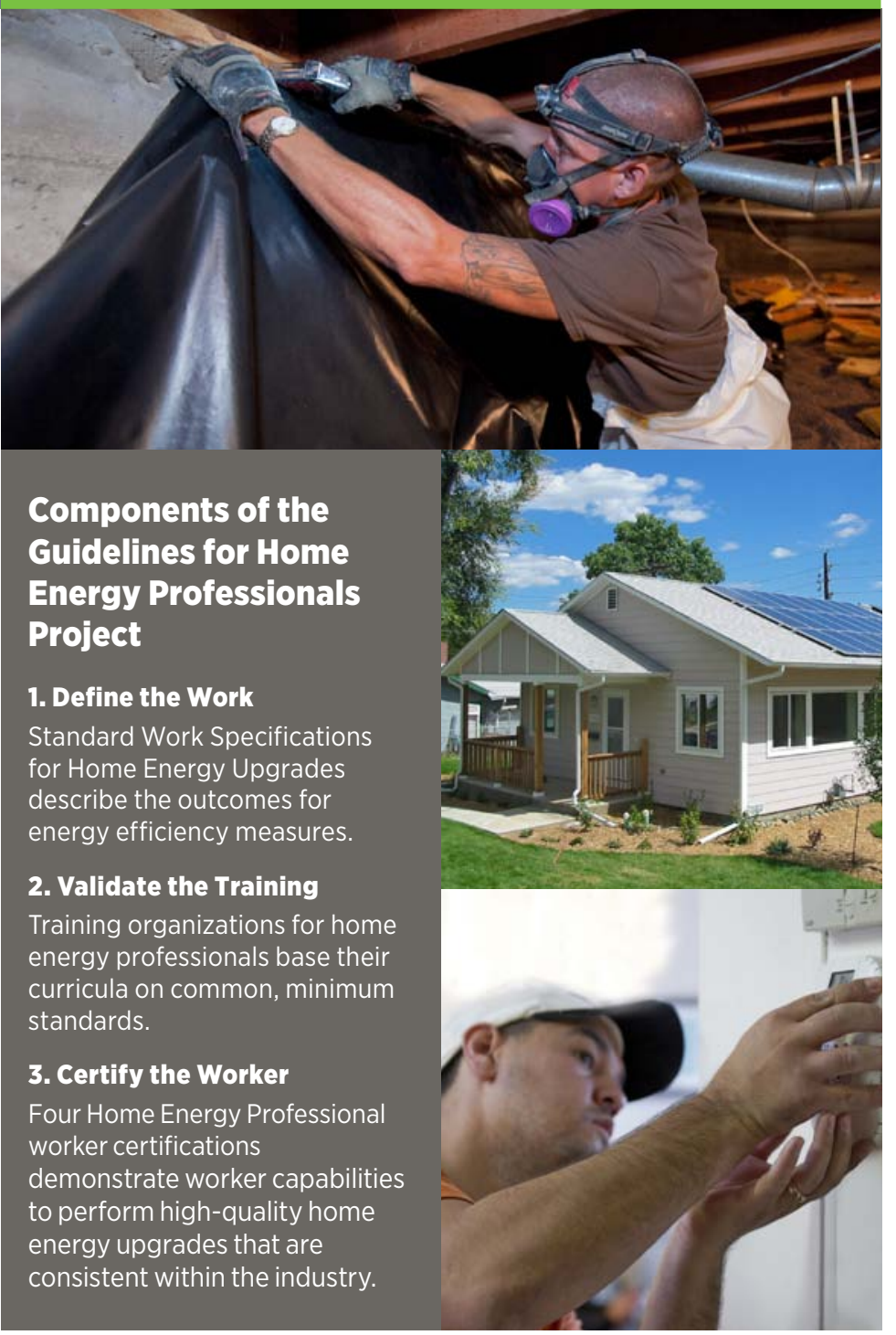

\section{Objectives for Efficiency Measures}

The Standard Work Specifications address a complete set of energy efficiency measures that comprise home energy upgrades, including energy audits, air sealing, insulation, and more. Safe work practices, relevant codes, and U.S. Environmental Protection Agency (EPA) Healthy Indoor Environment Protocols for Home Energy Upgrades are also included in the document.

Rather than attempting to prescribe specific actions, materials, or techniques, the Standard Work Specifications identify desired outcomes for each energy efficiency measure, listing the minimum set of requirements necessary to meet those outcomes. For example, trainers of home energy efficiency and weatherization workers might want to refer to specific sections of the Standard 


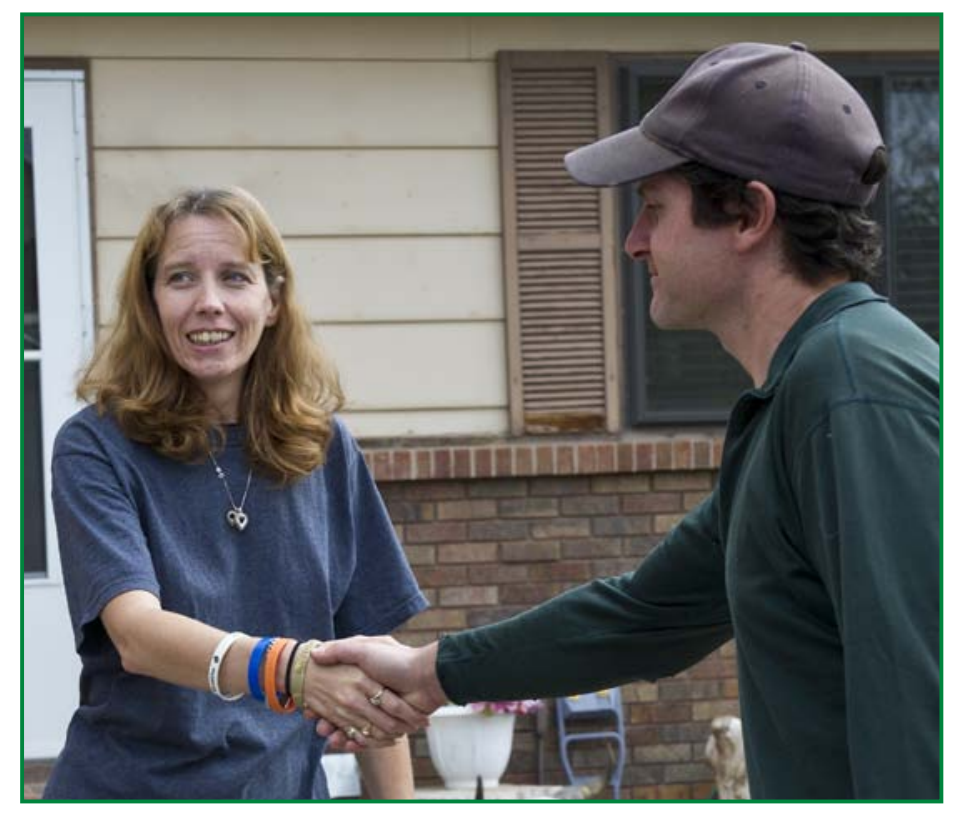

Home performance contractors that use the SWS can easily explain to homeowners how they will benefit from energy upgrades to their homes. Photo by Dennis Schroeder, NREL/PIX 22092

Work Specifications in their classrooms or curricula. Some weatherization and home energy efficiency programs might want to create field manuals complete with diagrams and instructions, based on the agreed-upon specifications. The innovation and necessity of the home performance and weatherization industry will determine how the quality of home energy upgrades will continue to evolve into the future.

By setting these overall objectives, the Standard Work Specifications remove potential confusion among industry participants about what constitutes a quality energy efficiency upgrade. Using a measurable set of outcomes as a baseline, all participants can work toward common high-quality goals that they can use, in turn, to communicate with the industry's customer base of homeowners.
"In my opinion, the creation of the Standard Work Specifications (SWS) will stand out as a pivotal moment in the history of both the weatherization and home performance industries. The SWS are the yardstick to which all of our past work can be measured, and the bar in which the future of our industry can strive to exceed."

- Chris Baker, Energy Training \& Technical Assistance Coordinator at the SouthWest Building Science Training Center

\section{Professional Training and Certification}

In addition to addressing quality work through the Standard Work Specifications, the Guidelines for Home Energy Professionals project also addresses worker training and certification. These additional efforts allow everyone in the industry (including homeowners) to speak the same language, share the same expectations, and have the same understanding of weatherization and home energy upgrade work.

Read more about the project at wip.energy.gov/retrofit_guidelines.html.
Energy Efficiency \& Renewable Energy 